\title{
An Investigation for Steganalysis in Color Images
}

Samah F. Aziz

samah.fakhri@uohamdaniya.edu.iq

AlHamdaniya University, Mosul, Iraq
Ahmed S. Nori

ahmed.s.nori@uomosul.edu.iq

College of Computer Science and

Mathematics, University of Mosul, Iraq

\section{Received on: 22/06/2011}

Accepted on: 16/08/2011

\section{ABSTRACT}

With science developing and techniques used in Information hiding, there are another techniques wall together for Steganalysis.

Steganography is considered as the new and the complementary system of Cryptography that took a long time in transferring secret and important messages through the networks Internet. Then there was the emergence of what complements Steganography as a science that analysis and discover the content of the secret messages and this science is Steganalysis.

This study tackled and manifested the ideas of analysis processes that can be followed to interpret the secret messages and discovering them either by means of knowing about their existence only or the capability of extracting them in full.

The work relied on two important technologies; the first is called the Support Vector Machine (SVM) and the second is called Fisher Linear Discriminator (FLD). The SVM technology has been used with the blind application idea while FLD has been used with the blind and non-blind application ideas using colored images which are PNG and BMP.

Results proved the high efficiency of the two technologies in detecting the image that includes the secret messages and comparisons were varied between the two technologies in terms of detection rate, fault and the execution time.

Keywords: Steganography, Steganalysis, SVM, FLD, PNG, BMP.

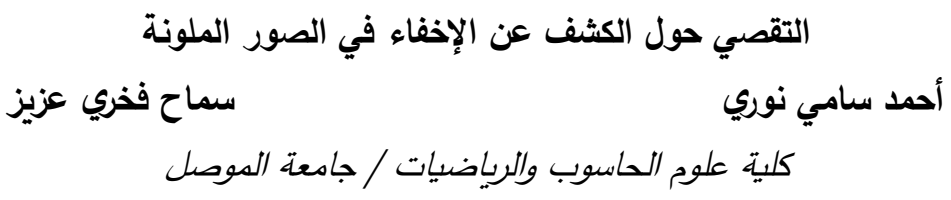

تاريخ قبول البحث: 2011/08/16

تاريخ استلام البحث: 2011/06/22

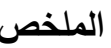

مع تطور العلم والتقنيات المستخدمة في تقنيات إخفاء المعلومات (Information Hiding)، هنالك تقنيات تتطور بموازاتها في تحليل الإخفاء (Steganalysis).

يعد الإخفاء (Steganography) النظام الجديد والمتمم لعلم التشفير الذي استغرق وقتا طويلا في تتاقل الرسائل

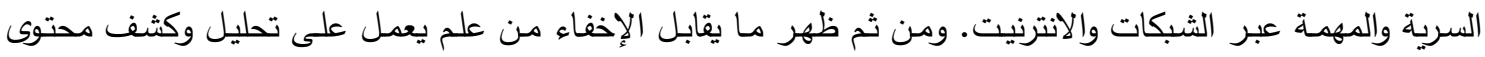

الرسائل السرية فكان علم كثف الإخفاء (Steganalysis).

Support ) SVM اعتمد العمل على تقنيتين مهمتين في عمليات التصنيف تسمى الأولى آلة المتجه الداعلماء

(ctor MachineV.e

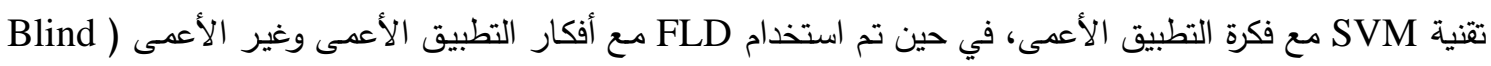
(and Non-Blind الكفاءة العالية للتقيتين في الكثف عن الصورة التي تحوي الرسائل السرية وتفاوتت المقارنات مابين التقنيتين من ناحية نسبة الكثف ومقدار الخطأ وزمن التنفيذ. الكلمات المفتاحية: الاخفاء، تحليل، آلة المتجه الداعم، مميز فشر الخطي، PNG، BMP. 
خـلال السنوات الماضـية، أصـبح علم أمن المعلومـات محل اهتمـام الكثير مـن الباحثين الذين تحـاول جهودهم أن تتوصل إلى حلول وتتنيات جديدة لضمان حماية المعلومات التي ترسل وتستقبل عبر الانترنيت دون

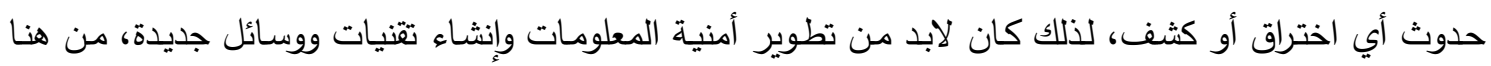

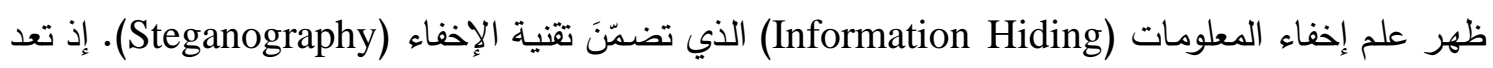
تقنية الإخفاء من طرائق الحماية التي تجعل الاتصال غير مرئي عن طريق إخفاء رسائل معينة داخل غطاء معين.

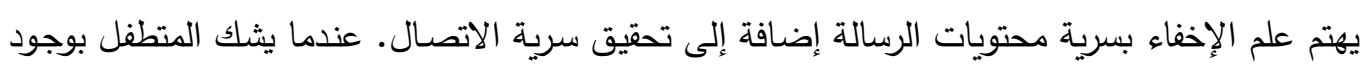

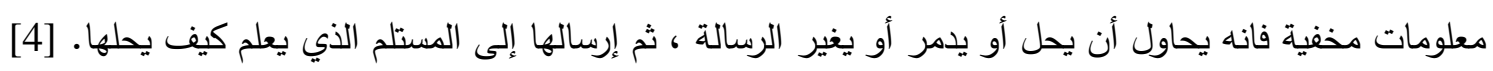

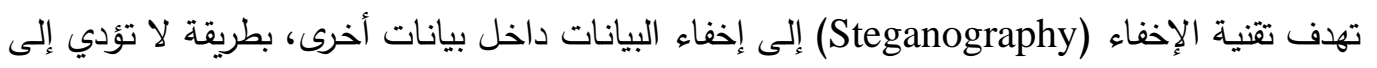
التأثير في هذه الأخيرة، بحيث لا تثير أي شبهة أو شك قد يؤديان إلى كثف المعلومات المخفية.

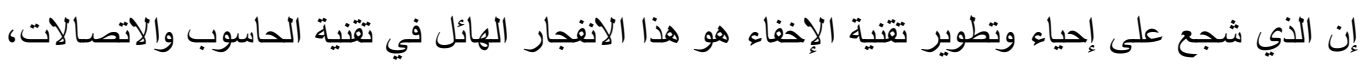

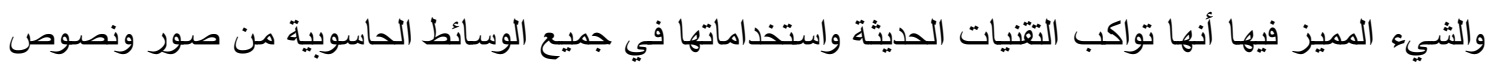
وصوت وفيديو ... الخ. إذ أصبحت أمنية المعلومات من الموضوعات الحساسة والمهمة جدا في حياة البشر خاصة بعد انتشار الحكومات الالكترونية في معظم دول العالم. [2] القصد من هذه المقدمة، أنه مع تطور العلم والأساليب المستخدمة في الإخفاء فهنالك أساليب تتطور

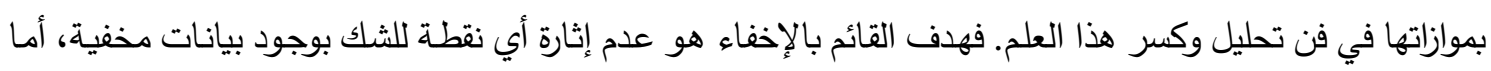

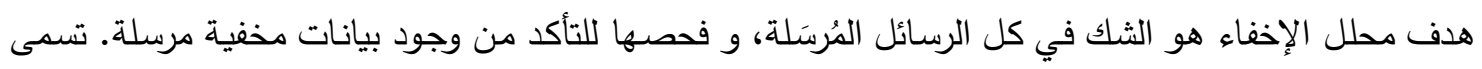

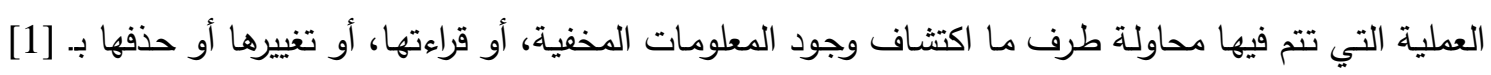
.Steganalysis 2- 2 - الدراسات السابقة

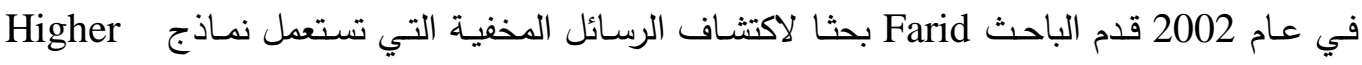
حيث يصف طريقة جديدة لاكتشاف الرسائل المخفية عن طريق استخدام Wavelet Statistics النماذج في الصور الطبيعية مع اعتماد تقنية Fisher Linear Discriminant Analysis للتمييز بين الصور

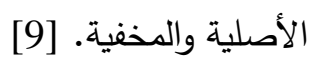

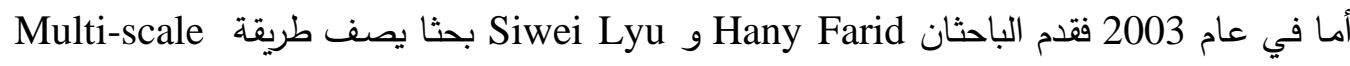
Wavelet Decomposition

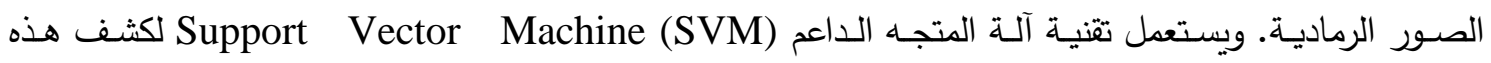

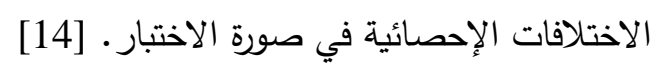

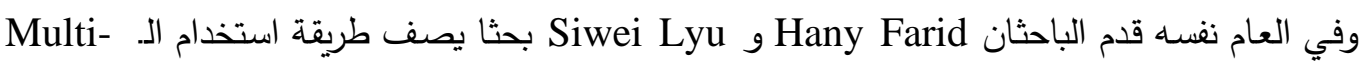
scale Wavelet Decomposition (QMF) Quadrature Mirror Filters Fisher Linear Discriminant Analysis 
قدم الباحثون Wiang و Wong و Munon و Wu مام 2005 تقنية Steganalysis جديدة في صور

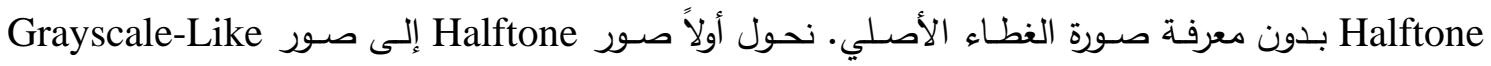
باستخدام Low-Pass Filtering, ومن ثم تحلل باستخدام Quadrature Mirror Filters للحصول علدول على

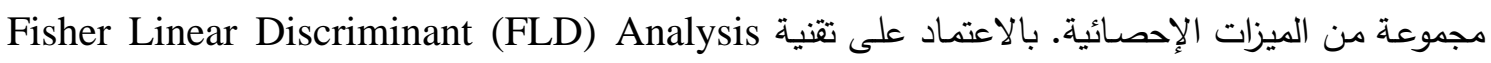

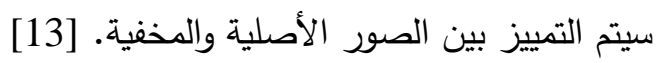

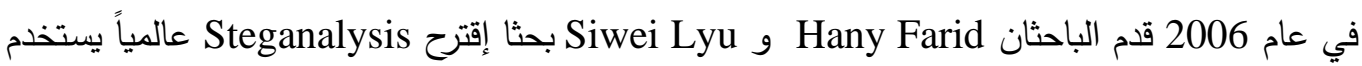
Higher Order Statistics إحصائيات الصورة. مع اعتماد One Class and Multi Class SVM للتصنيف. من مساوئ الخوارزمية عدد الميزات المطلوب لتدريب SVM يكون عالياً, وذلك يتطلّب وقت أكثر لاكتشاف صورة stego. [17]

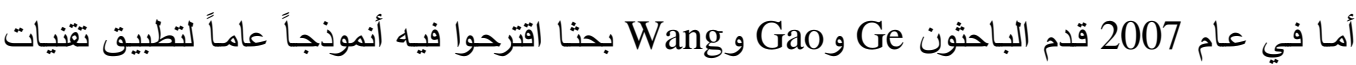
تعليم الآلة لكثف المعلومات المخفية باستخدام تقنية إخفاء في البت الأقل أهمية. والاعتماد على مصنف إحصائي لتصنيف الصور • [11]

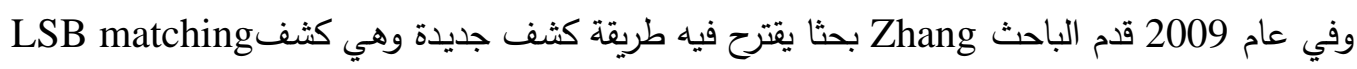

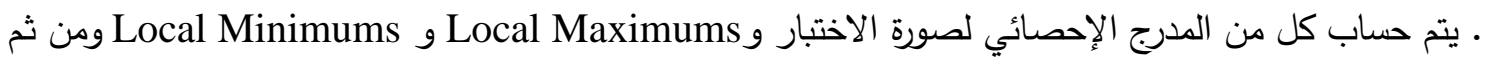

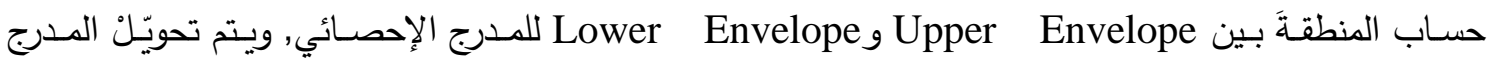
الإحصائي باستخدام DWT لحساب الفرق بين كل من Local Maximums و Local Minimums و و Neighbors [19] 3- الإخفاء وتحليل الإخفاء

إخفاء المعلومات ضمن وسط إلكتروني يتطلّب تعديلات على خصائص ذلك الوسط التي قد تُقدم شكلاً

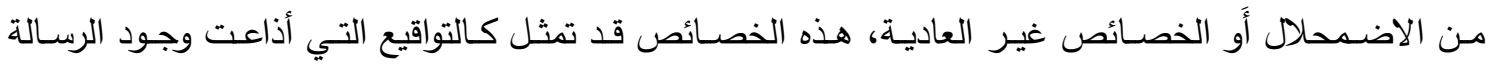
المُضَمَّنة، وهذا يضعف الغرض من الإخفاء.

تحليل الإخفاء Steganalysis هو علم اكتشاف الرسائل المخفية باستخدام الإخفاء. من الناحية الأخرى,

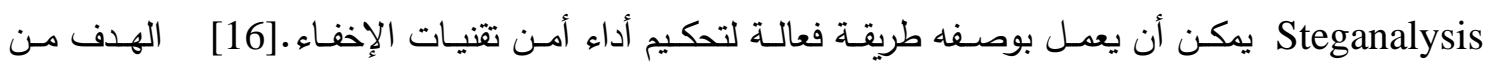
Steganalysis أن يكتثف وجود الرسالة السرية في الأجمسام (objects) ويُميز الأجسام بالرسـالة السرية مِن فين فئن

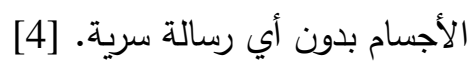
الهجمات والتحليل على المعلومات المخفية قد تأخذ عدة أثكال: اكتثاف أو انتزاع، أو تعطيل أَو تحطيم

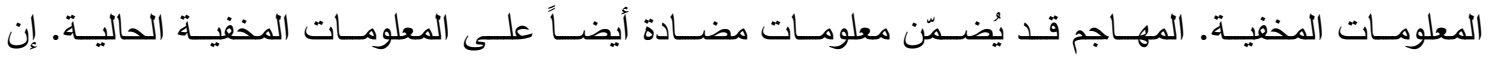

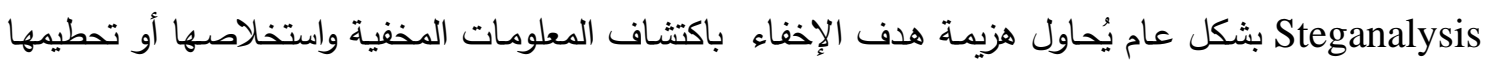

Steganalyst عملية كثف الإخفاء تتم من قبل جهة أخرى غير المرسل والمستقبل وهو محلل الإخفاء وهو الثخص الذي يطبق تحليل الإخفاء في محاولة لكثف وجود المعلومات المخفية. [20] 4- الهجمات على الإخفاء 
جَذب الإخفاء وتحليل الإخفاء الكثير من الانتباه حول العالم في المستقبل القريب, وذلك لاهتمام البعض حضل

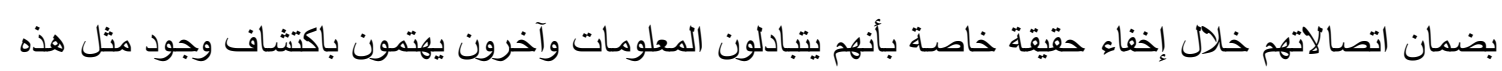

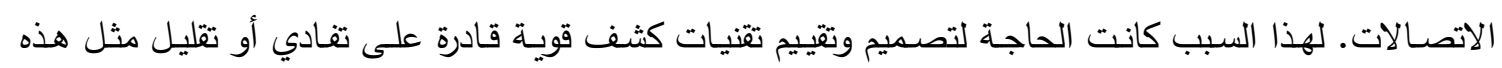

الأعمال. [15]

يُُكن أَن تكون الهجمات على الإخفاء بأنواع مختلفة تعتمد على الأسباب أَو الغرض من الهجوم ضد

بيانات_stego. والهجوم الناجح هو الذي يشمل كثف المحتوى المخفي. يمكن تصنيف أنواع الهجمات على الإخفاء إلى صنفين عامّين هما:

1 1 الهجوم السلبي Passive Attacks

هذا الهجوم يكثف حضور أو غياب الرسالة السرية المتضمنة في بيانات_stego أو تحديد نوع تضمين الخوارزمية المستخدمة. والمهاجم قادر على اعتراض البيانات فقط. وتتضمن:

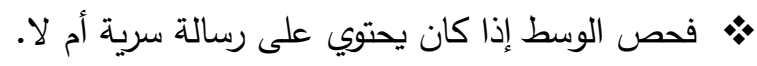
• • استخلاص الرسالة السرية إذا كان بالإمكان استخلاصها. • ثمطيم الرسالة السرية.

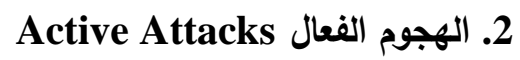

تخمين أو انتزاع خصائص الرسالة أو خوارزمية التضمين لتحوير بيانات_stego من اجل تدمير البيانات المضمنة. يحاول محلل الإخفاء تخمين وانتزاع الرسالة السرية بدون تحطيمها, أي انه قادر على معالجة البيانات.

وبشكل عام، تُصنف تقنيات تحليل الإخفاء إلى صنفين وكما يأتي:

1. تحليل الإخفاء المستهدف عargeted Steganalysis

وتعني عملية الكثف عن خوارزمية إخفاء معروفة. إذ يُكن أن يكثف الرسالةً السرية أو حتى يُخمّن نسبة التضمين مع معرفة خوارزمية الإخفاء.

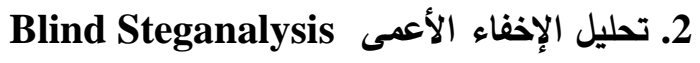

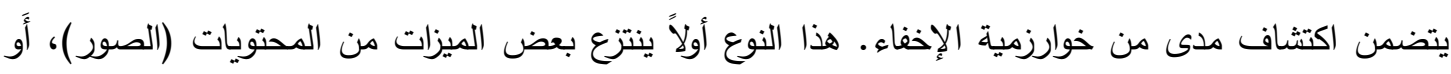

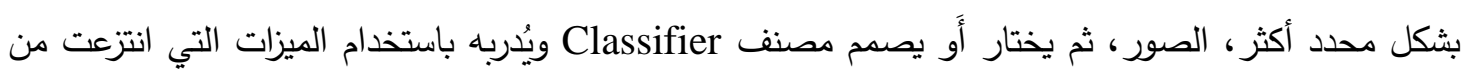

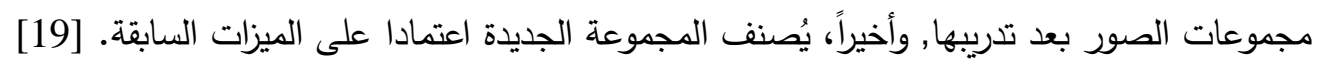

5- فكرة البحث الأساسية

نتيجة الإخفاء المستمر وظهور تقنيات إخفاء جديدة تم اقتراح نظام للتقصي عن تحليل الإخفاء (Steganalysis)

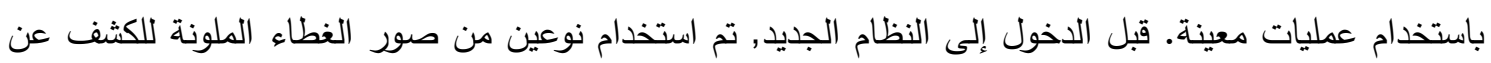

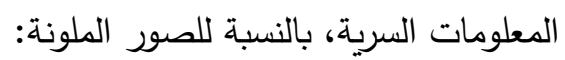
• • م الامتداد BMP تم الإخفاء باستخدام برنامج (S-Tools V 4.0).

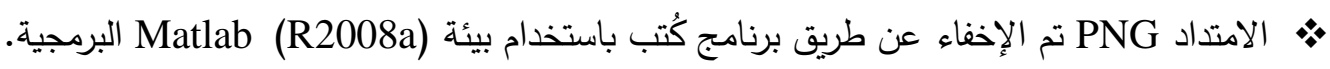
يتم تطبيق النظام المقترح على مرحلتين رئيستين هما كالآتي: 
1. عملية استخلاص الخواص Features Extraction.

2. عملية تطبيق الخوارزمية الجديدة، عن طريق تقنية آلة المتجه الداعم (SVM)، وتقنية مميز فيشر الخطي

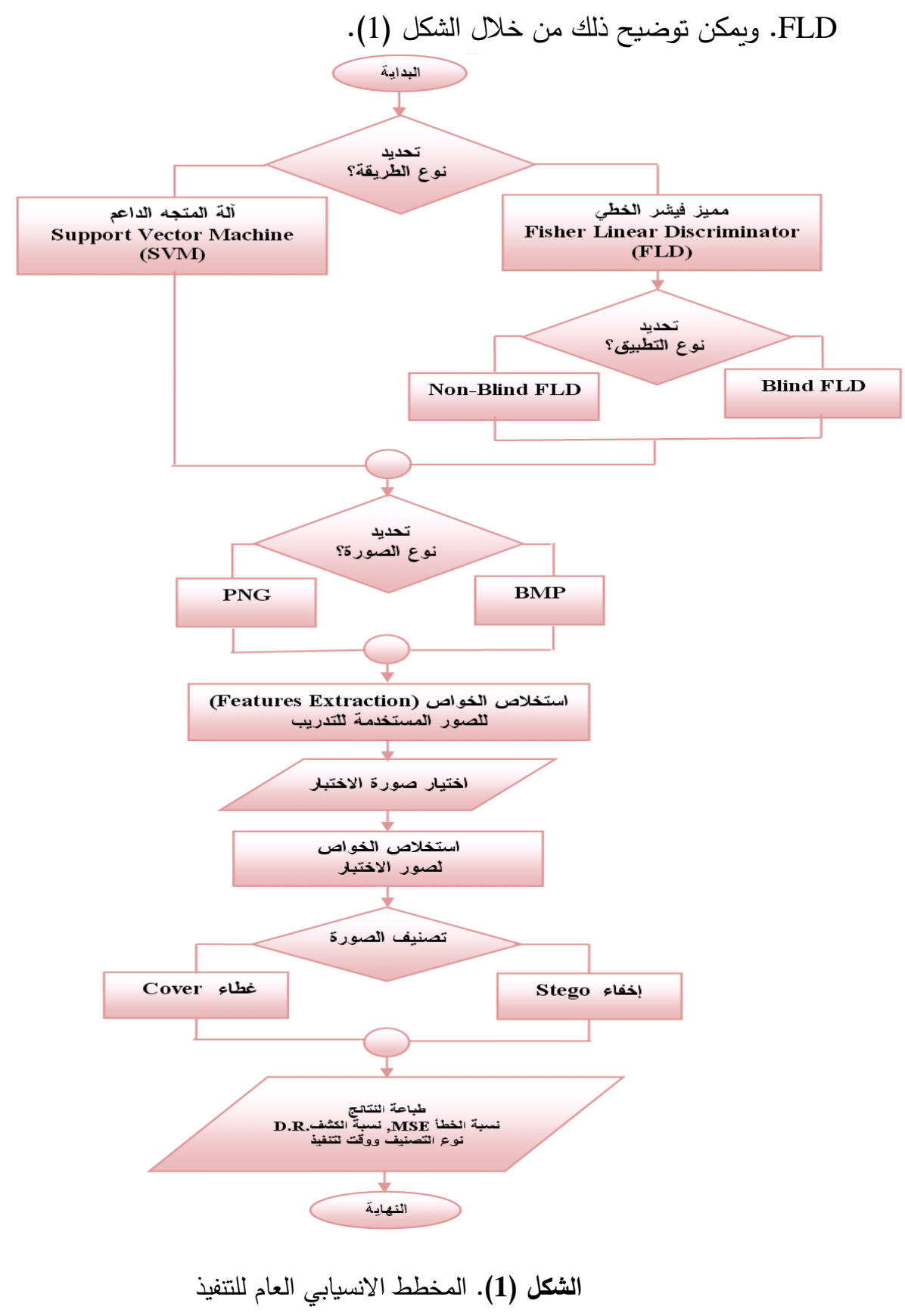


Features Extraction 6- استخلاص الخواص:

تعتمد عملية استخلاص الخواص من الصور الملونة بالامتدادين (PNG , BMP) على بناء أنموذج

إحصائي يستخدم فيه المدرج الإحصائي (Histogram) وتحويل فوريير (Discrete Fourier Transform)

وذلك للحصول على متجهات الخواص (Features Vectors).

$$
\text { الإدخال: صورة الاختبار (BMP) أو (PNG). }
$$

الإخراج: متجهات الخواص (Features Vectors)، وعددها (24) متجهاء.

أما الخطوات فتشمل:

1. تحليل الصورة إلى مستوياتها اللونية الثلاثة (RGB).

2. بناء مدرج إحصائي لكل مستوى لوني (1Dimension ) (R , G , B)

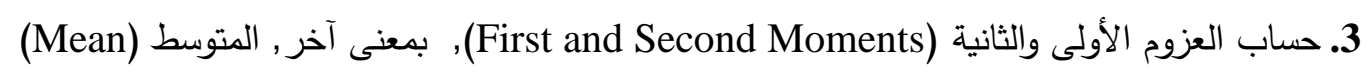

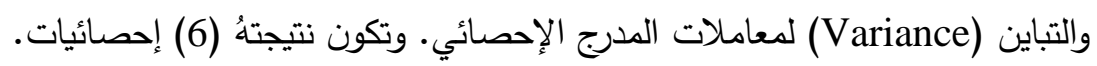
4. تطبيق تحويل فوريير (Discrete Fourier Transform) على المدرج الإحصائي ولكل مستوى لوني (RGB)

5. حساب العزوم (الأولى، الثانية، الثالثة والرابعة) بمعنى آخر , المتوسط, التباين، الالتواء (Skewness) و (Kurtosis) لمعاملات تحويل فوريير (DFT). وهنا ينتج (12) إحصائية.

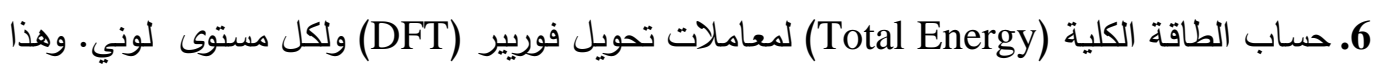
ينتج (3) إحصائيات. 7. حساب متوسط الفرق بين معاملات المدرج الإحصائي و تحويل فوريير المنفصل لكل مستوى لوني، هذا ينتج أيضا (3) إحصائيات. 8. تجميع الإحصائيات في متجهات الخواص لتصبح (24) إحصائية.

Support Vector Machine آلة المتجه الداعم

قُّمت هذه التقنية في عام (1992) من قبل الباحث (Vapnik) [6]، وهي عبارة عن خوارزمية تعلم عن

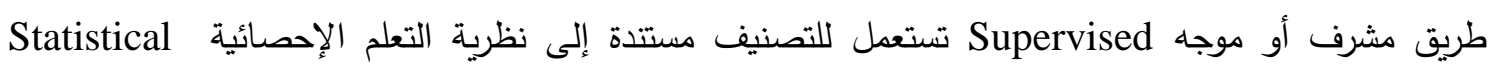
[11].Learning Theory تقنية آلة المتجه الداعم (SVM) كسبت مؤخراً أهمية في حقل تعلم الآلة وتصنيف الأنماط, إذ أنها

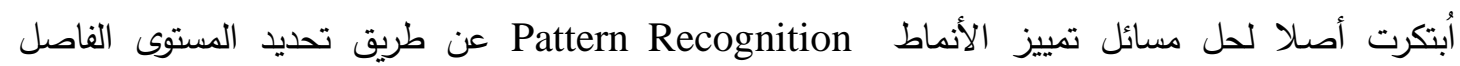
Hyperplane مستوى فاصل Hyperplane للبيانات المراد فصلها وتصنيفها على صنفين.

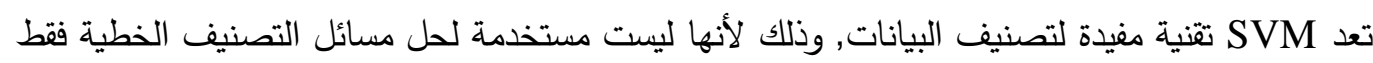

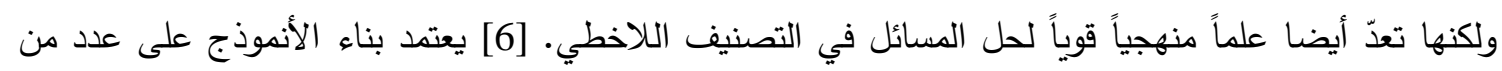

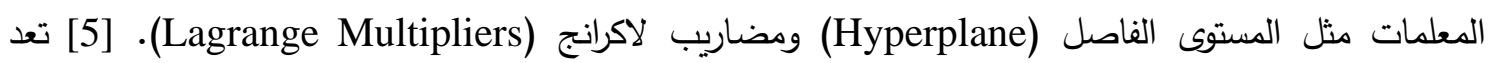
عملية استخلاص الخواص للحصول على المتجهات خطوة أساسية وأولية للبدء في تقنية آلة المتجه الداعم SVM. 
بعدها يتم تدريب التقنية على مجموعة البيانات الناتجة من استخلاص الخواص للحصول على الأوزان المثالية،

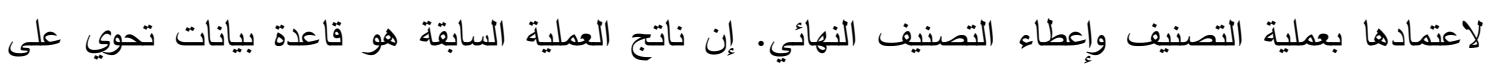
مجموعة من متجهات الخواص التي تم الحصول عليها لمجموعة من الصور المستخدمة لتدريب التقنية.

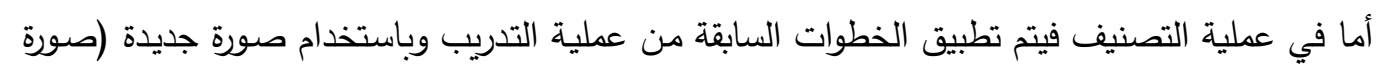

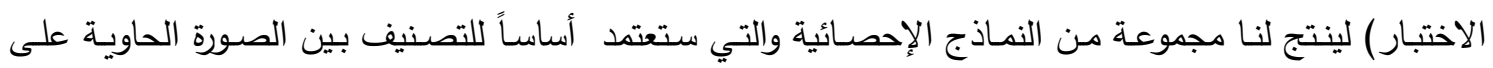
معلومات سرية والصورة غير الحاوية تلك المعلومات.

8isher Linear Discriminator (FLD) تقنية مميز فيشر الخطي عبارة عن طريقة تصنيف إحصائية، إذ قُم في عام (1936) من قبل الباحث فيشر (Fisher)، [3] وهو تقنية تصنيف قياسية مستخدمة على نطاق واسع ومثبتة في الكثير من تطبيقات العالم الحقيقي، ومنها (تمييز [18] الأنماط)

تهدف الطريقة إلى إيجاد أفضل إسقاط خطي مثالي (Optimal Linear Projection) بين مجموعتين أو أكثر في عملية التصنيف, والحصول على أعلى تمييز بين المجتمعات وذلك يكون بجعل نسبة التباين بين

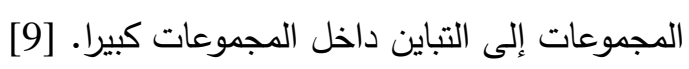
تقوم فكرة هذه الخوارزمية على تدريب مجموعة متجهات الخواص الناتجة من عملية استخلاص الخواص

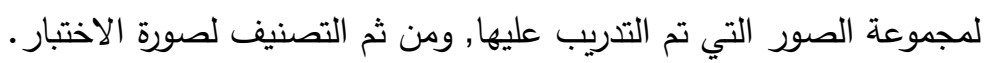
-9 - (النتائج أُخذت مجموعة صور ملونـة ذات أبعاد 400 ×600 وبالامتدادين BMP و PNG. تم اعتماد برنامج

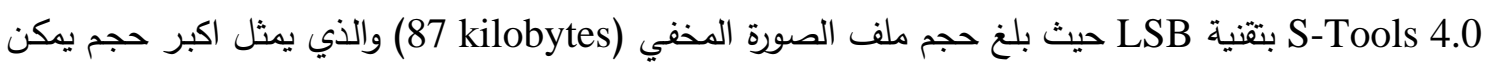
إخفاءه في صور BMP (400×600) باستخدام برنامج 4-Tools 4.0. أما إخفاء نص بحجم 62786 حرفا في صور PNG كان باستخدام LSB. وكان عدد الصور المستخدمة للتدريب (100 غطاء/ 100 إخفاء). أما عدد الصور المستخدمة في الاختبار (100 غطاء الصور المعاء 100 إخفاء).

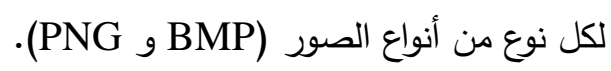
تم التطبيق لتتنية آلة المتجه الداعم على مرحلتين وهي:

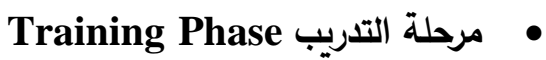

شَملت هذه المرحلـة تدريب تقنيـة آلـة المتجـه الـداعم SVM على مجموعتين مـن متجهات الخـواص:

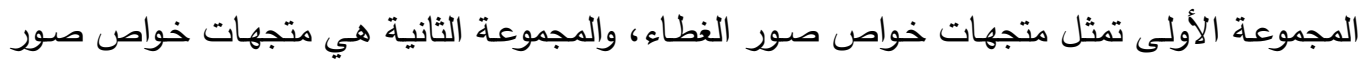

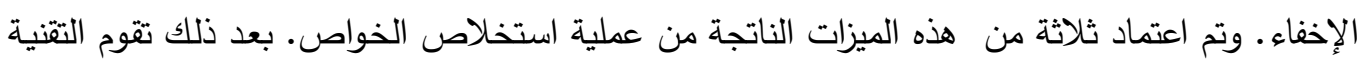
بعملية التصنيف وإعطاء التصنيف النهائي.

Testing Phase مرحلة الاختبار مرحلة الاختبار شملت تطبيق تقنية آلة المتجه الداعم SVM على مجموعة جديدة من 
متجهات الخـواص لمجموعـة جديدة من صـور الغطاء والإخفاء. في عملية التصـيف تؤخذ النمـاذج

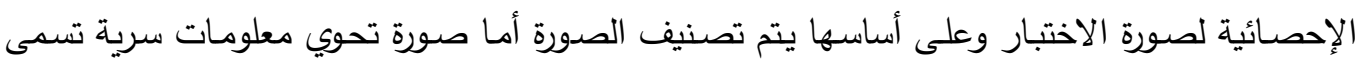

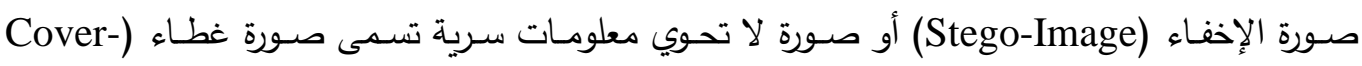
.(Image وكانت نتائج اختبار صور ملونة ذات امتداد BMP باستخدام تطبيق تقنية آلة المتجه الداعم SVM كما هو مبين في الجدول (1). (1) - (1) نسبة الكثف (DR) = عدد الصور المضمنة بصورة صحيحة / عدد الصور في الاختبار مقدار الخطأ (MSE) = $=\sum_{M, N}[\operatorname{stego-im}(m, n) \text { - cover-im }(m, n)]^{2} /\left(M^{*} N\right)$

$$
\begin{aligned}
& \text { M عدد الصفوف لصور الإدخال. } \\
& \text { N عدد الأعمدة الصور الإدخال. }
\end{aligned}
$$

\begin{tabular}{|c|c|c|c|c|c|}
\hline نوع التصنيف & زمن التنفيذ & مقدار الخطأ & 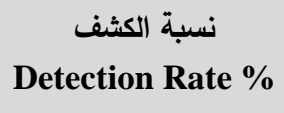 & اسم الصورة & ت \\
\hline cover & 0.25 & 0.03 & 91 & yellow bird & 1 \\
\hline cover & 0.17 & 0.08 & 95 & Coffee & 2 \\
\hline cover & 0.22 & 0.04 & 91 & blue car & 3 \\
\hline cover & 0.24 & 0.03 & 93 & sunflower & 4 \\
\hline cover & 0.59 & 0.09 & 97 & horse race & 5 \\
\hline cover & 0.27 & 0.05 & 92 & Flowers & 6 \\
\hline cover & 0.21 & 0.07 & 96 & Child & 7 \\
\hline cover & 0.25 & 0.05 & 92 & Natural & 8 \\
\hline cover & 0.35 & 0.05 & 96 & Bird & 9 \\
\hline cover & 0.20 & 0.06 & 95 & Girl & 10 \\
\hline stego & 0.26 & 0.02 & 92 & s-yellow bird & 1 \\
\hline stego & 0.23 & 0.05 & 93 & s-coffee & 2 \\
\hline stego & 0.26 & 0.07 & 94 & s-blue car & 3 \\
\hline stego & 0.22 & 0.04 & 93 & s-sunflower & 4 \\
\hline stego & 0.22 & 0.06 & 95 & s-horse race & 5 \\
\hline stego & 0.23 & 0.06 & 96 & s-flowers & 6 \\
\hline stego & 0.22 & 0.03 & 92 & s-child & 7 \\
\hline stego & 0.22 & 0.04 & 93 & s-natural & 8 \\
\hline stego & 0.40 & 0.08 & 96 & s-bird & 9 \\
\hline stego & 0.22 & 0.07 & 93 & s-girl & 10 \\
\hline
\end{tabular}

الجدول (1). نتائج تطبيق تقنية SVM على صور BMP الملونة (400 × 600)

ومن خلال ملاحظة الجدول (1) يتبين ما يأتي:

إن هذاك فرقا في المقاييس المستخدمة (نسبة الكثف, مقدار نسبة الخطأ، زمن التنفيذ) بحيث تراوحت نسب الكشف مابين (97-91)\% بالنسبة للصور ذات التصنيف (Cover)، في حين كانت النسب هي

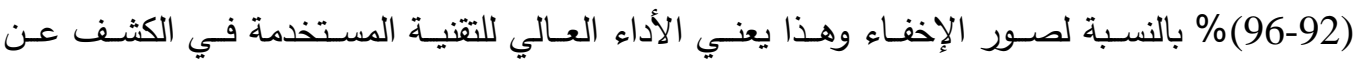
(Stego) (Cover)

وفيما يخص مقدار الخطأ نلاحظ أن النسبة تراوحت مابين (0.03 - 0.09) للصور المصنفة (Cover)،

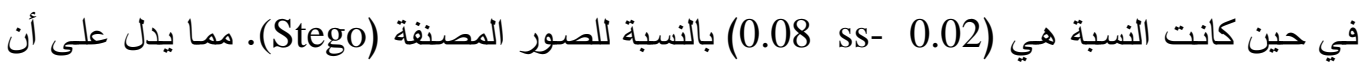
التقنية المستخدمة لا تملك مقدار خطأ ملحوظ. 


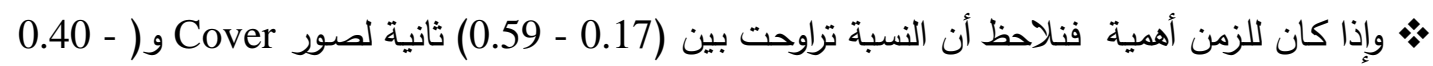

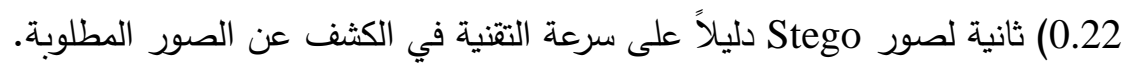

علما أن النتائج المثبتة في الجدول (1) كانت كلها صحيحة وبالنسبة للكثف عن الت الـ (Cover) و (Stego).

ولإثبات كفاءة التقنية SVM تم تطبيقها على أنموذج شائع الاستخدام وهو (PNG). أما النتائج فيمكن مشاهدتها

في الجدول (2). (2) (2)

الجدول (2). نتائج تطبيق تقنية SVM على صور PNG الملونة (400 × 600)

\begin{tabular}{|c|c|c|c|c|c|}
\hline نوع التصنيف & زمن التتفيذ & مقدار الخطأ & $\begin{array}{c}\text { نسبة الكشف \% } \\
\text { Detection Rate }\end{array}$ & اسم الصورة & ت \\
\hline cover & 0.06 & 0.28 & 73 & Balloon & 1 \\
\hline cover & 0.10 & 0.28 & 75 & Sea & 2 \\
\hline cover & 0.12 & 0.33 & 82 & Book & 3 \\
\hline cover & 0.12 & 0.21 & 68 & Girls & 4 \\
\hline cover & 0.09 & 0.31 & 79 & Tree & 5 \\
\hline cover & 0.09 & 0.20 & 73 & Snake and bird & 6 \\
\hline cover & 0.09 & 0.24 & 72 & flowers & 7 \\
\hline cover & 0.08 & 0.26 & 72 & pen & 8 \\
\hline cover & 0.08 & 0.23 & 72 & digital & 9 \\
\hline cover & 0.07 & 0.18 & 71 & cat & 10 \\
\hline stego & 0.12 & 0.28 & 74 & balloon & 1 \\
\hline stego & 0.05 & 0.25 & 74 & sea & 2 \\
\hline stego & 0.04 & 0.22 & 71 & book & 3 \\
\hline stego & 0.06 & 0.31 & 77 & girls & 4 \\
\hline stego & 0.05 & 0.28 & 74 & tree & 5 \\
\hline stego & 0.12 & 0.26 & 71 & Snake and bird & 6 \\
\hline stego & 0.05 & 0.22 & 71 & flowers & 7 \\
\hline stego & 0.05 & 0.25 & 73 & pen & 8 \\
\hline stego & 0.07 & 0.27 & 77 & digital & 9 \\
\hline stego & 0.09 & 0.23 & 72 & cat & 10 \\
\hline
\end{tabular}

فيما يخص نتائج تطبيق تقنية FLD بنوعيها الأعمى (Blind FLD) و الغير الأعمى (Non-Blind FLD) تم

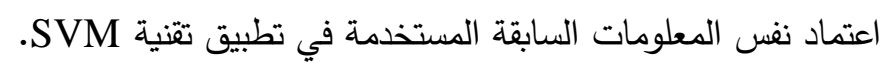

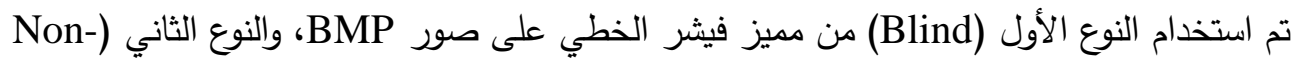

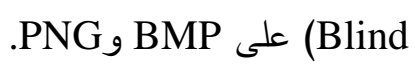
وفيما يخص نتائج اختبار الصور الملونة بامتداد BMP باستخدام تقنية مميز فيشر الخطي الأعمى كما

هو مبين في الجدول (3). 


\begin{tabular}{|c|c|c|c|c|}
\hline \multicolumn{5}{|c|}{ تقنية BLDd FLD على صور BMP الملونة (400 × 600) } \\
\hline \multicolumn{5}{|c|}{ صور غطاء } \\
\hline $\begin{array}{c}\text { نسبة الكشف Detection Rate } \\
\text { \% }\end{array}$ & التصنيف & زمن التنفيذ & اسم الصورة & $ت$ \\
\hline \multirow{10}{*}{$100 \%$} & Cover & 0.12 & yellow bird & 1 \\
\hline & Cover & 0.13 & coffee & 2 \\
\hline & Cover & 0.13 & blue car & 3 \\
\hline & Cover & 0.14 & sunflower & 4 \\
\hline & Cover & 0.13 & horse race & 5 \\
\hline & Cover & 0.14 & flowers & 6 \\
\hline & Cover & 0.13 & child & 7 \\
\hline & Cover & 0.13 & natural & 8 \\
\hline & Cover & 0.14 & bird & 9 \\
\hline & Cover & 0.11 & girl & 10 \\
\hline \multicolumn{5}{|c|}{ صور إخفاء } \\
\hline \multirow{10}{*}{$100 \%$} & Stego & 0.11 & s-yellow bird & 1 \\
\hline & Stego & 0.11 & s-coffee & 2 \\
\hline & Stego & 0.10 & s-blue car & 3 \\
\hline & Stego & 0.13 & s-sunflower & 4 \\
\hline & Stego & 0.11 & s-horse race & 5 \\
\hline & Stego & 0.13 & s-flowers & 6 \\
\hline & Stego & 0.14 & s-child & 7 \\
\hline & Stego & 0.11 & s-natural & 8 \\
\hline & Stego & 0.14 & s-bird & 9 \\
\hline & Stego & 0.10 & s-girl & 10 \\
\hline
\end{tabular}

وتم تطبيقت التقنيـة FLD نـوع (Non-Blind) على أنمـوذج صـور (BMP). وكانـت نتـائج الاختبـار

باستخدام تقنية مميز فيشر الخطي غير الأعمى وكما هو مبين في الجدول (4) و(5).

Non-Blind FLD الجدول (4). نتائج اختبار صور غطاء ملونة ذات امتداد BMP باستخدام

\begin{tabular}{|c|c|c|c|c|}
\hline $\begin{array}{c}\text { نسبة الكشف \% } \\
\text { Detection Rate \% }\end{array}$ & تصنيف صورة & زمن التنفيذ & صورة غطاء & ت \\
\hline \multirow{10}{*}{$100 \%$} & cover & 0.14 & yellow bird & 1 \\
\hline & cover & 0.12 & coffee & 2 \\
\hline & cover & 0.12 & blue car & 3 \\
\hline & cover & 0.13 & sunflower & 4 \\
\hline & cover & 0.15 & horse race & 5 \\
\hline & cover & 0.16 & flowers & 6 \\
\hline & cover & 0.12 & Child & 7 \\
\hline & cover & 0.14 & natural & 8 \\
\hline & cover & 0.14 & Bird & 9 \\
\hline & cover & 0.14 & Girl & 10 \\
\hline
\end{tabular}


الجدول (5). نتائج اختبار صور إخفاء ملونة ذات امتداد BMP باستخدام Non-Blind FLD

\begin{tabular}{|c|c|c|c|c|}
\hline $\begin{array}{c}\text { نسبة الكشف \% } \\
\text { Detection Rate \% }\end{array}$ & تصنيف صور & زمن التنفيذ & صورة إخفاء & ت \\
\hline \multirow{10}{*}{$100 \%$} & stego & 0.14 & s-yellow bird & 1 \\
\hline & stego & 0.12 & s-coffee & 2 \\
\hline & stego & 0.12 & s-blue car & 3 \\
\hline & stego & 0.13 & s-sunflower & 4 \\
\hline & stego & 0.15 & s-horse race & 5 \\
\hline & stego & 0.16 & s-flowers & 6 \\
\hline & stego & 0.12 & s-child & 7 \\
\hline & stego & 0.14 & s-natural & 8 \\
\hline & stego & 0.14 & s-bird & 9 \\
\hline & stego & 0.14 & s-girl & 10 \\
\hline
\end{tabular}

وبالنسبة لنتائج اختبار صورة PNG باستخدام هذه التقنية بنوعها غير الأعمى كما هو مبين في الجدول (6) (7).

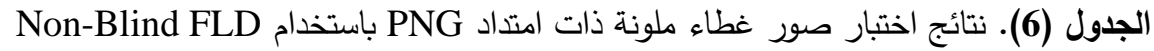

\begin{tabular}{|c|c|c|c|c|}
\hline $\begin{array}{c}\text { نسبة الكشف \% } \\
\text { Detection Rate \% }\end{array}$ & تصنيف صورة & زمن التنفيذ & صورة غطاء & ت \\
\hline \multirow{10}{*}{$80 \%$} & stego & 0.13 & Color & 1 \\
\hline & cover & 0.09 & Sun & 2 \\
\hline & cover & 0.10 & Green & 3 \\
\hline & stego & 0.09 & gray car & 4 \\
\hline & cover & 0.09 & digital & 5 \\
\hline & cover & 0.09 & apple & 6 \\
\hline & cover & 0.09 & yellow car & 7 \\
\hline & cover & 0.10 & player & 8 \\
\hline & cover & 0.09 & motor & 9 \\
\hline & cover & 0.09 & Pen & 10 \\
\hline
\end{tabular}

الجدول (7). نتائج اختبار صور إخفاء ملونة ذات امتداد PNG باستخدام Non-Blind FLD

\begin{tabular}{|c|c|c|c|c|}
\hline $\begin{array}{c}\text { نسبة الكثف \%etection Rate \% } \\
\text { \% }\end{array}$ & تصنيف صور & زمن التنفيذ & صورة إخفاء & 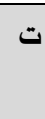 \\
\hline \multirow{10}{*}{$80 \%$} & cover & 0.13 & s-color & 1 \\
\hline & stego & 0.09 & s-sun & 2 \\
\hline & stego & 0.10 & s-green & 3 \\
\hline & cover & 0.09 & s-gray car & 4 \\
\hline & stego & 0.09 & s-digital & 5 \\
\hline & stego & 0.09 & s-apple & 6 \\
\hline & stego & 0.09 & s-yellow car & 7 \\
\hline & stego & 0.10 & s-player & 8 \\
\hline & stego & 0.09 & s-motor & 9 \\
\hline & stego & 0.09 & s-pen & 10 \\
\hline
\end{tabular}

ومن خلال ملاحظة الجداول (6) و (7) يتبين ما يلي:

نلاحظ أن الزمن تراوح مـابين (0.09 - 0.13) ثانية لصور الغطاء والإخفاء وهذا بسبب اختيار

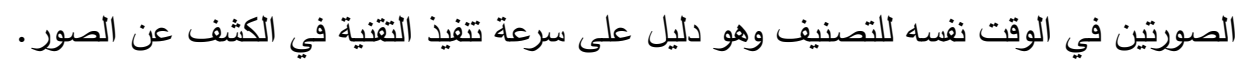

ما استطاعت التقنية أن تتبت مدى سرعتها من خلال النتائج التي حصلنا عليها, وهذا يدل على كفاءة التقنية 
ويعرض الثكل (2). نموذجا من الصور التي أجريت عليها اختبارات الكثف بامتداد BMP وPNG.

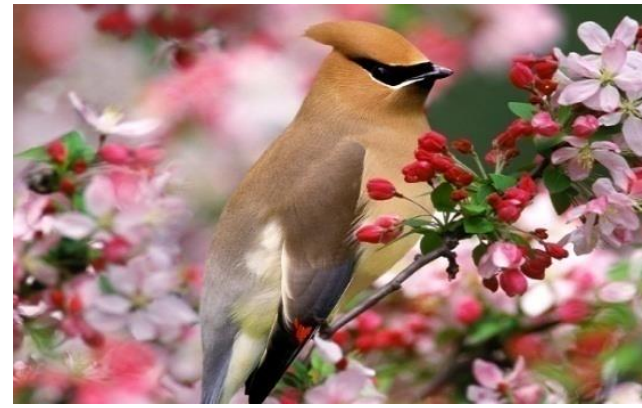

ب- بورة أصلية بامتداد PNG

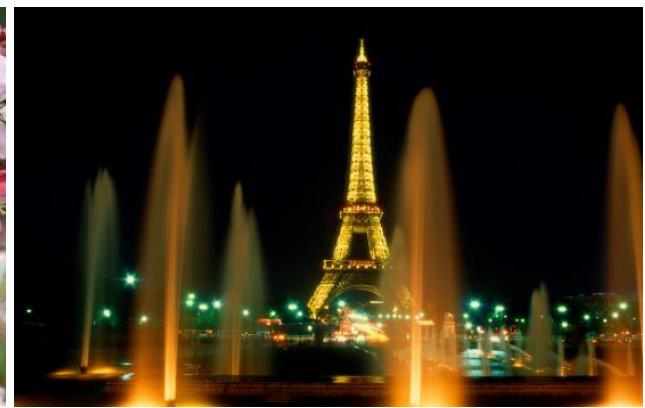

أ- صورة أصلية بامتداد BMP

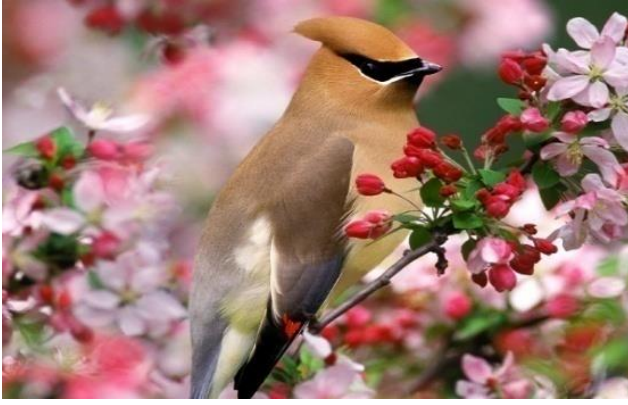

د د دورة غطاء بامتداد PNG

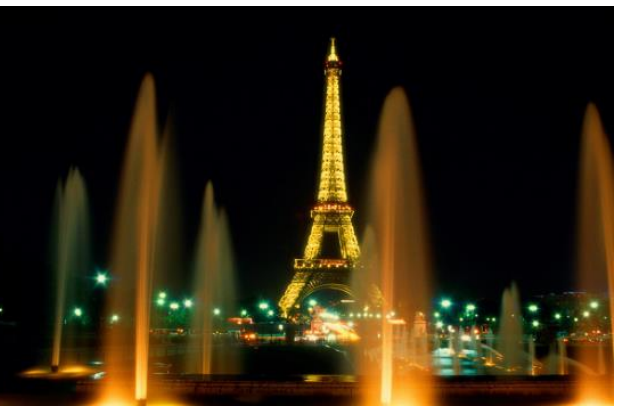

ج- صورة غطاء بامتداد BMP

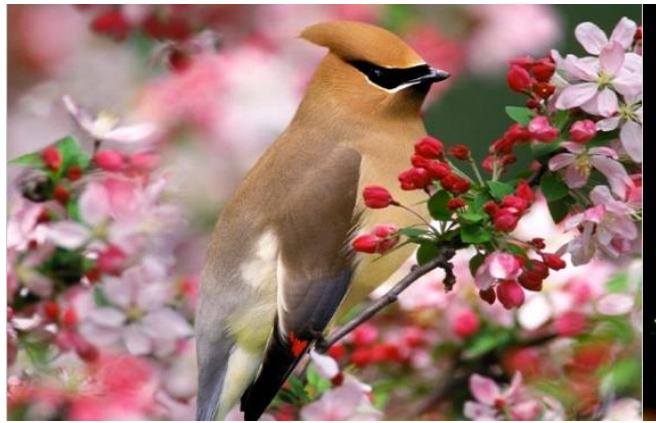

و - صورة إخفاء بامتداد PNG باستخدام LSB

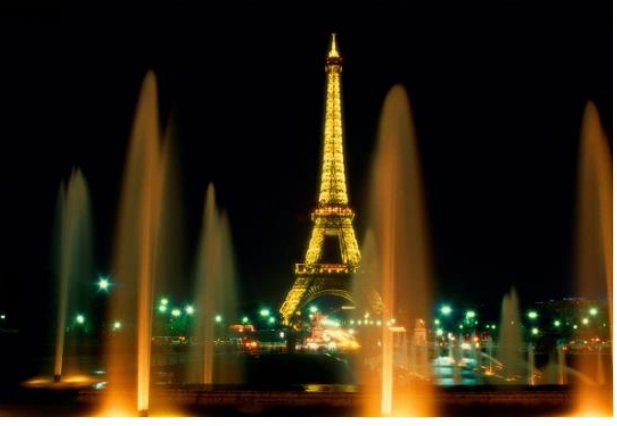

هـ -- صـورة إخفـاء بامتـداد BMP باسـتخدام S-Tools برنامج إخفاء

الثكل (2). نموذج من الصور التي أجريت عليها اختبارات كثف صورة

وبمقارنة النتائج الخاصة بالجداول (1) و (2) مع فقرة الدراسات السابقة (5,2) من حيث نسبة الكثف لتقنية

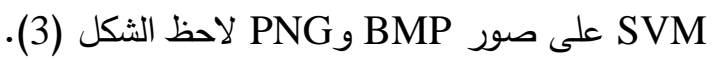
أما ما يخص المقارنة النتائج الخاصة بالجداول (1) و (2) من حيث مقدار نسبة الخطأ لتقنية SVM ملى SVM BMP و PNG فلاحظ الثكل (4). 


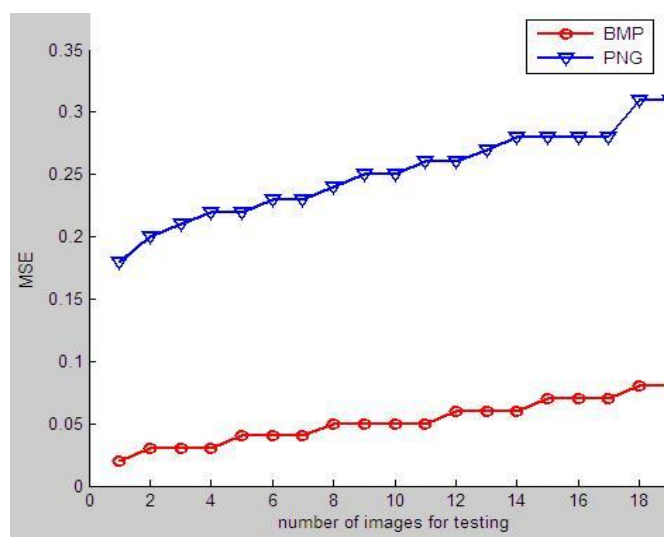

الثكل (4). يوضح مقارنة قيمة مقدار الخطأ لتقنية

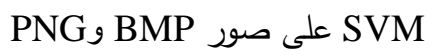

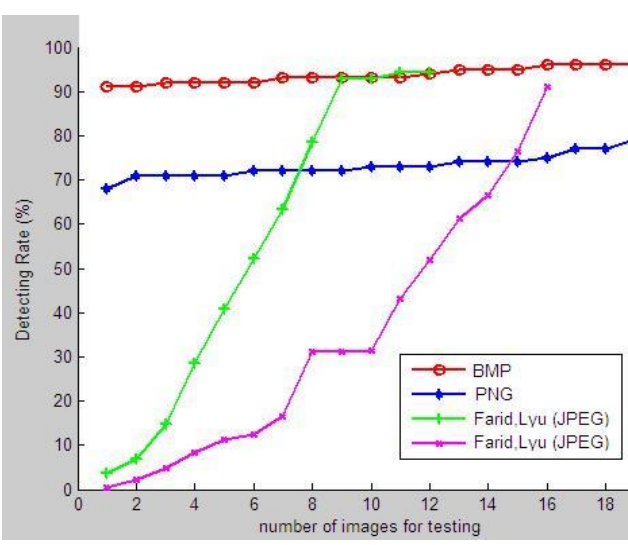

الثكل (3). يوضح نتائج مقارنة الدراسات السابقة (5,2) لنسبة الكثف باستخدام تقنية

SVM

وفيما يخص مقارنة النتائج الخاصـة بالجداول (1) و (2) من حيث زمن التنفيذ لتقنية SVM

BMP و PNG فلاحظ الثكل (5).

وبالنسبة لمقارنة النتائج الخاصة بالجداول (1) و (3) من حيث زمن التنفيذ لتقنية SVM blind و SLD

blind على صور BMP لاحظ الثكل (6).

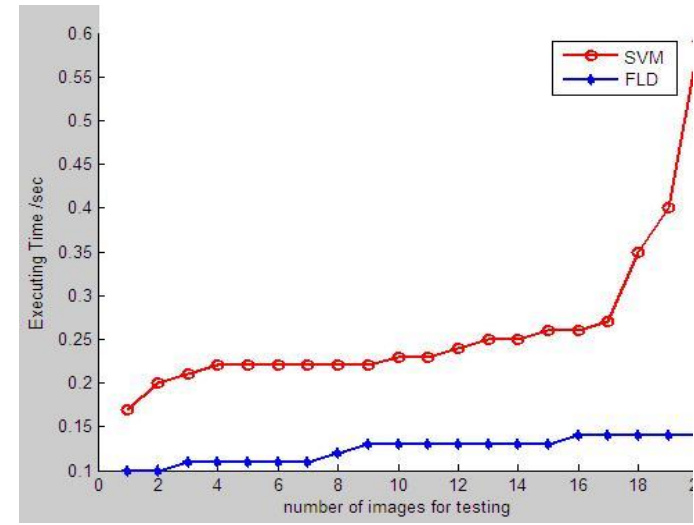

الثكل (6). يوضح مقارنة مقدار وقت

التنفيذ لتقنية SVD و SVlind

على صور BMP

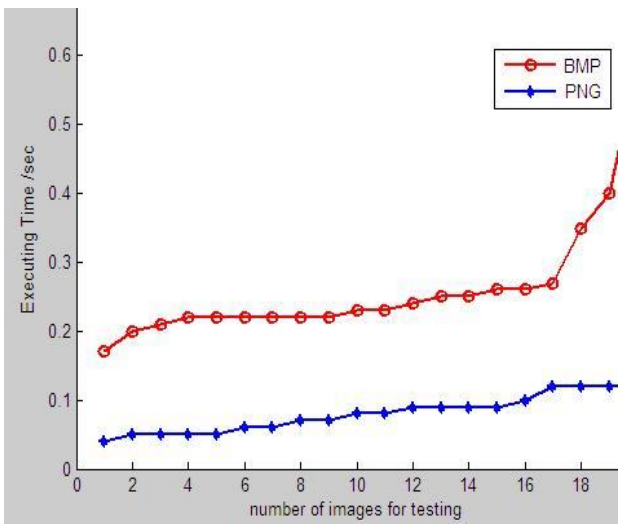

الثكل (5). يوضح مقارنة مقدار وقت

التتفيذ لتقنية SVM على صور PNG,

FLD non- أما فيما يخص مقارنة النتائج الخاصة بالجداول (4) و (5) و (6) و (7) من حيث زمن التنفيذ لتتنية blind على صور BMP و PNG لاحظ الثكل (7). 


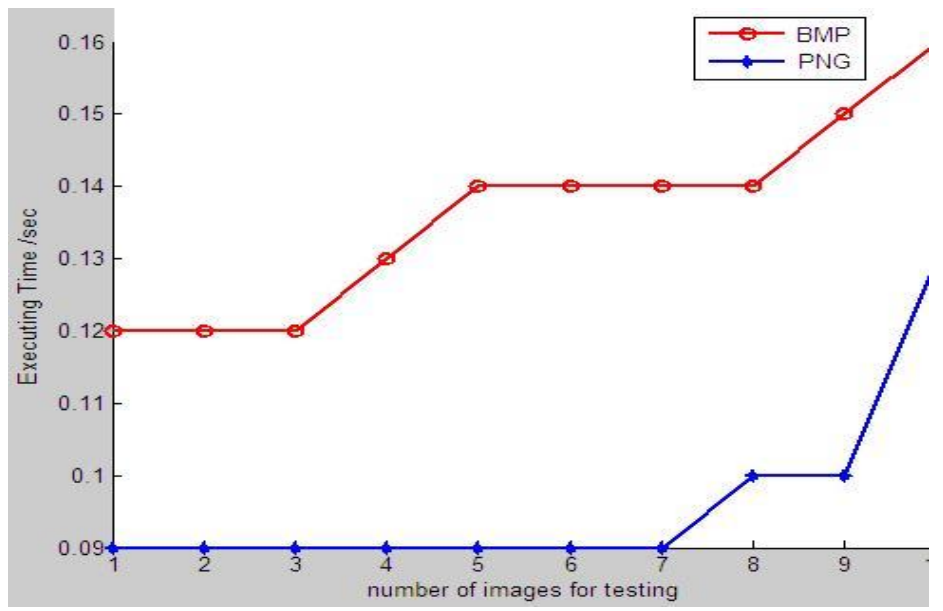

الثكل (7). يوضح مقارنة مقدار وقت التنفيذ لتقنية FLD non-blind على صور BMP وPNG

وفيما يخص مقارنة النتائج من حيث مقدار نسبة الكثف لتقنية FLD non-blind على صور BMP و PNG لاحظ الثكل (8). وبمقارنة النتائج الخاصة بتقنية FLD مع فقرة الدراسات السابقة (7,1) من حيث نسبة الكثف لاحظ الثكل (9).

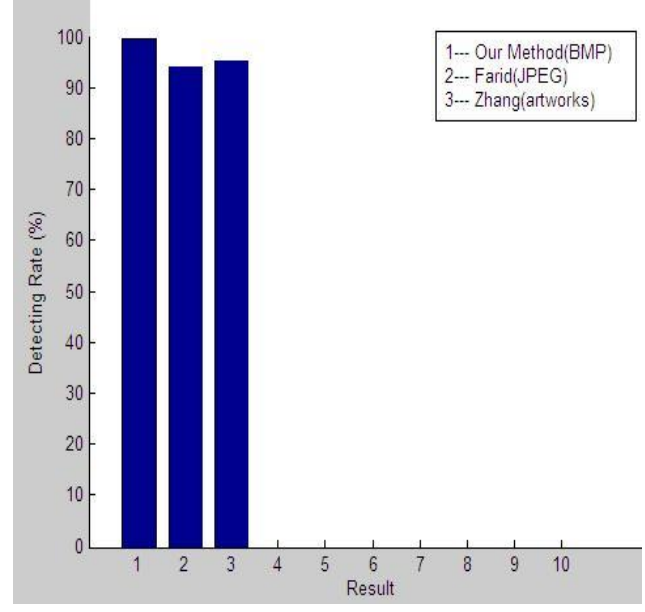

الثكل (9). يوضح نتائج مقارنة الدراسات السابقة (7,1) لنسبة الكثف باستخدام FLD تقنية

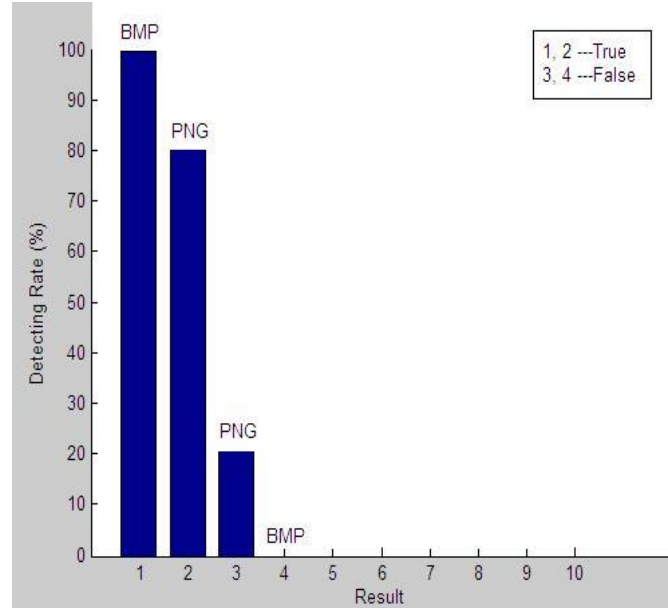

الثكل (8). يوضح مقارنة مقدار نسبة الكثف لتقنية FLD non-blind على صور BMP و PNG

10- الاستنتاجات Conclusions

$$
\text { من الأمور الأساسية لكل محلل هو: }
$$

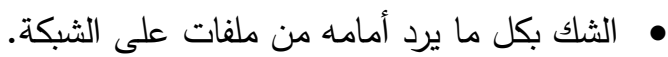

• فكرة عمل محلل الإخفاء هو بطبيعة الحال اعقد وله درجة من الصعوبة أكثر من فكرة عمل الإخفاء.

• • لا بد للمحلل أن يكون مطلعاً وملماً بأكثر الطرائق المستخدمة في الإخفاء ولاسيما الحديثة منها.

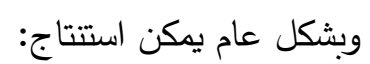


1. للتوصل إلى عملية كثف صحيحة ودقيقة لا بد من توفر إحدى المعلومات التالية Secret Cover)

.(Algorithm ,Message

2. هناك علاقة طردية مابين كمية البيانات المخفية وتقنية الكشف.

3. أثبتت النتائج جودة تقنية آلة المتجه الداعم SVM في الكثفة عنف عن الإخفاء في الصور بالاعتماد على

$$
\text { مقياس نسبة الكثف التي تجاوزت }
$$

4. فكرة التدريب العشوائي لمجموعة التدريب تكون أفضل لان نسبة الكثف تتغير حسب التدريب بدلا من تثبيت مجموعة التدريب, وكلما زادت أحجام مجموعة التدريب كان الكثف أفضل.

5. امتازت تقنية مميز فيشر الخطي (FLD) بأداء عالٍ وسرعة كبيرة في الكثف نظرا لقلة الوقت المستغرق في التدريب.

6. أعطت تقنية FLD مع أفكار (Blind) و Non-Blind) نتائج جيدة جدا وكانت الأفضلية لـ (Non)

.(Blind

7. التحكم في اختيار الخواص (الميزات الإحصائية) المستخدمة من الصورة أعطى قابلية اكبر للكثف عن الإخفاء.

8. وكمقارنة بين التقنيتين فيما بينهما (وبشكل عام) كانت الأفضلية لتقنية SVM على FLD من ناحية

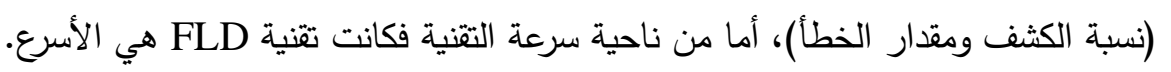

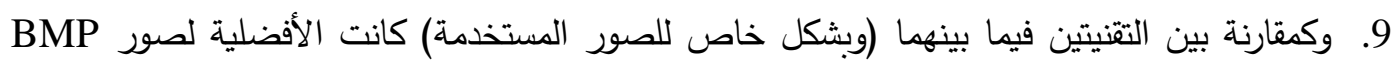

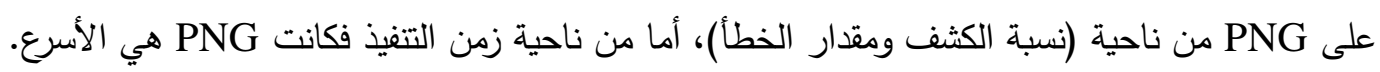




$$
\begin{aligned}
& \text { المصادر } \\
& \text { برزنجي، فوزي، 2008، "إخفاء البيانات داخل الصورة"، جامعة السليمانية، العراق. } \\
& \text { fowzibarazenji@yahoo.com www.boosla.com } \\
& \text { الحمامي، علاء حسين والحمامي، محمد علاء، 2008، "إخفاء المعلومات: الكتابة المخفية والعلامة } \\
& \text { المائية"، إثراء للنشر والتوزيع، الثارقة. } \\
& \text { الراوي، عمر فوزي صالح، 2004، "استخدام الدالة التمييزية في السيطرة النوعية مع تطبيق على ولادات }
\end{aligned}
$$

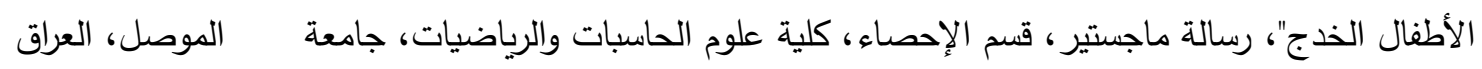

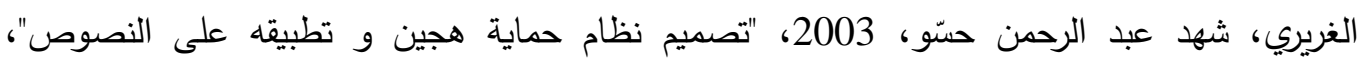

$$
\begin{aligned}
& \text { رسالة ماجستير، قسم علوم الحاسبات، كلية علوم الحاسبات والرياضيات، جامعة الموصل، العراق. } \\
& \text { قاسم، عمر صابر ، 2009، "تطبيق التقنيات الذكائية في المعلوماتية الحياتية "، أطروحة دكتوراه، قسم } \\
& \text { الرياضيات، كلية علوم الحاسبات والرياضيات، جامعة الموصل، العراق. }
\end{aligned}
$$

[6] Ahmad A., Khalid M. and Yusof R., "Machine Learning Using Support Vector Machines", Universiti Teknologi Malaysia.

\{marzuki,rubiyah\}@utmkl.utm.my

[7] Cheng J., Kot A., and et.al., ICASSP 2005, "STEGANALYSIS OF BINARY TEXT IMAGES", Nanyang Technological University, Singapore.

[8] Du Q., 2007 IEEE,"Modified Fisher's Linear Discriminant Analysis for Hyperspectral Imagery", Senior Member, IEEE GEOSCIENCE AND REMOTE SENSING LETTERS, VOL. 4, NO. 4, OCTOBER 2007.

[9] Farid H., 2002, " DETECTING HIDDEN MESSAGES USING HIGHERORDER STATISTICAL MODELS", Department of Computer Science, Dartmouth College, Hanover.

farid@cs.dartmouth.edu

[10] Farid H. and Lyu S., 2003, " Higher-order Wavelet Statistics and their Application to Digital Forensics", IEEE Workshop on Statistical Analysis in Computer Vision (in conjunction with CVPR).

[11] Ge S., Gao Y. and Wang R., 2007 ACM, " Least Significant Bit Steganography Detection with Machine Learning Techniques ",National Laboratory for Novel Software Technology Nanjing University 210093 Nanjing, Jiangsu, China, geshengeorge@gmail.com gaoy@nju.edu.cn R.wang@massey.ac.nz

[12] Ivanciue, O., (2007), "Applications of support Vector Machines in Cjemistry", Wiley-VCH, John Wiley \& Sons, Volume 23 pp. 291400

[13] Jiang M., Wong E., Memon N. and Wu X., ICASSP 2005, "Steganalysis of Halftone Images", IEEE Int'l Conf on Acoustics, Speech, and Signal Processing, Philadelphia, PA.2005.

[14] Lyu S. and Farid H., Springer-Verlag Berlin Heidelberg 2003, " Detecting Hidden Messages Using Higher-Order Statistics and Support Vector Machines", Dartmouth College, Hanover, USA 
[15] Rocha A., and Goldenstein S., (2008), " Steganography and Steganalysis in Digital Multimedia: Hype or Hallelujah?", RITA, Vo. 15, No. 1, pp.83-110.

[16] Shi Y., Xuan G., and et.al., "Steganalysis Based on Moments of Characteristic Functions Using Wavelet Decomposition, Prediction-Error Image, and Neural Network", New Jersey Institute of Technology, Newark, NJ, USA, Tongji University, Shanghai, China.

[17] Siwei Lyu and Hany Farid, 2006, "Steganalysis using Higher-Order Image Statistics", IEEE Transaction on Information Forensics and Security, vol. 1, pp. 111-119, 2006.

[18] Yang J., Jina Z., Yang Y. and Frang A., 2003, "Essence of kernel Fisher discriminant: KPCA plus LDA", Department of Computer Science, Nanjing University of Science and Technology, Nanjing.

[19] Zhang J., Hu Y. and Yuan Z., ACADEMY PUBLISHER 2009, "Detection of LSB Matching Steganography using the Envelope of Histogram", Guangdong University of Business Studies, Guangzhou P.R. China, JOURNAL OF COMPUTERS, VOL. 4, NO. 7, JULY 2009.

Zhangjundan123@yahoo.com.cn okhyp@yahoo.com.cn

[20] http://coeia.edu.sa/ar/asuurance-awarness/articles/57-cryptography-andsteganography-and-pki/557-steganography.html 